\title{
Factorizing regular graphs
}

Thomassen, Carsten

Published in:

Journal of Combinatorial Theory. Series B

Link to article, DOI:

10.1016/j.jctb.2019.05.002

Publication date:

2019

Document Version

Peer reviewed version

Link back to DTU Orbit

Citation (APA):

Thomassen, C. (2019). Factorizing regular graphs. Journal of Combinatorial Theory. Series B, 141, 343-351. https://doi.org/10.1016/j.jctb.2019.05.002

\section{General rights}

Copyright and moral rights for the publications made accessible in the public portal are retained by the authors and/or other copyright owners and it is a condition of accessing publications that users recognise and abide by the legal requirements associated with these rights.

- Users may download and print one copy of any publication from the public portal for the purpose of private study or research.

- You may not further distribute the material or use it for any profit-making activity or commercial gain

- You may freely distribute the URL identifying the publication in the public portal

If you believe that this document breaches copyright please contact us providing details, and we will remove access to the work immediately and investigate your claim. 


\title{
Factorizing regular graphs
}

\author{
Carsten Thomassen \\ Department of Applied Mathematics and Computer Science, \\ Technical University of Denmark, DK-2800 Lyngby, Denmark
}

March 18, 2019

\begin{abstract}
Every 9-regular graph (possibly with multiple edges) with odd edge-connectivity $>5$ can be edge-decomposed into three 3 -factors. If Tutte's 3-flow conjecture is true, it also holds for all 9-regular graphs with odd edge-connectivity 5 , but not with odd edge-connectivity 3 . It holds for all planar 2-edge-connected 9-regular graphs, an equivalent version of the 4-color theorem for planar graphs. We address the more general question: If $G$ is an $r$-regular graph, and $r=k q$ where $k, q$ are natural numbers $>1$, can $G$ be edge-decomposed into $k q$-factors? If $q$ is even, then the decomposition exists trivially. If $k, q$ are both odd, then we prove that the decomposition exists if $G$ has odd edgeconnectivity (size of smallest odd edge-cut) at least $3 k-2$, which is satisfied if the odd edge-connectivity is at least $r-2$. If $q$ is odd and $k$ is even, then we must require that $G$ has an even number of vertices just to guarantee that $G$ has a $q$-factor. If we want a decomposition into $q$-factors, then we also need the condition that, for any partition of the vertex set of $G$ into two odd parts, there must be at least $k$ edges between the parts. We prove that the edge-decomposition into $q$-factors is always possible if $G$ has an even number of vertices and the edge-connectivity of $G$ is at least $2 k^{2}+k$.
\end{abstract}

Keywords: graph factors

$\operatorname{MSC}(2000): 05 \mathrm{C} 70,05 \mathrm{C} 40,05 \mathrm{C} 20$ 


\section{Introduction}

The 4-color theorem for planar graphs is equivalent to the statement that every planar 2-edge-connected 3-regular graph can be edge-decomposed into three 1-regular graphs. This is also equivalent to the statement that every planar 2-edge-connected 9-regular graph can be edge-decomposed into three 3-regular graphs. To see that the latter implies the former, replace each vertex of degree 3 by a triangle in which each edge has multiplicity 4 . (Since each newly added multi-triangle does not contain a 3-factor, every 3-factor of the new graph must contain an original edge attached to the triangle, which in turn shows that each of the decomposed three 3 -factors contains exactly one such edge. So, these three 3 -factors give three 1 -factors of the original graphs.) In theorem 1 below we prove the latter version using the 4-color theorem.

While the former version does not extend to nonplanar graphs as shown by the Petersen graph (and all other snarks) we point out that the latter extends if we impose a condition on the odd edge-connectivity which is the size of a smallest odd edge-cut. Odd edge-connectivity at least 3 is necessary for the decomposition and is sufficient in the planar case but will not suffice in general as shown by the parenthetical remark above. We prove that, if Tutte's 3-flow conjecture holds, then odd edge-connectivity 5 will suffice. As a consequence of the weak 3-flow conjecture (now a theorem) odd edgeconnectivity 7 suffices.

Using the weak version of Jaeger's circular flow conjecture [4], [5] we then prove the general factorization result described in the abstract. As a consequence we prove some edge-decomposition results with constraints on the minimum (respectively maximum) degrees. A particularly strong decomposition result is obtained when $r$ is odd and divisible by 3: If $G$ is an $r$-regular, $(r-3)$-edge-connected graph of even order, where $r$ is odd and divisible by 3 , and $r=r_{1}+r_{2}+\cdots+r_{q}$ is any partition of $r$ with all parts $r_{i} \geq 2$, then $G$ can be edge-partitioned into subgraphs $G_{1}, G_{2}, \ldots, G_{q}$ such that $G_{i}$ is an $r_{i}$-factor for $i=1,2, \ldots, q$. 


\section{9-regular planar graphs and the 4-color the- orem}

The terminology and notation are the same as in [13] which are essentially the same as in [1], [6], [11]. In the present paper, however, a graph may have multiple edges (but no loops).

We now use the 4-color theorem to prove that every 2-edge-connected 9-regular planar graph has an edge-decomposition into three 3-factors. More generally, every 2 -edge-connected $3 q$-regular planar graph has an edge-decomposition into three $q$-factors.

This follows from the following even more general result. We formulate it in terms of edge-colorings with colors $1,2,3$. If $v$ is a vertex, then $c_{i}(v)$ denotes the number of edges of color $i$ incident with $v$.

Theorem 1 Let $G$ be a 2-edge-connected planar graph. Then $G$ has an edgecoloring in colors 1,2,3 such that, for each vertex $v$, two of $c_{1}(v), c_{2}(v), c_{3}(v)$ are equal, and the third differs from the two others by one of $0,2,-2$.

Proof of Theorem 1: Suppose (reductio ad absurdum) that Theorem 1 is false, and let $G$ be a counterexample such that

(i) the number of blocks of $G$ is minimum, and, subject to (i),

(ii) the number of vertex-degrees not divisibly by 3 is minimum, and, subject to (i),(ii),

(iii) the sum of vertex-degrees $>3$ is minimum, and, subject to (i), (ii), (iii),

(iv) the number of edges of $G$ is minimum.

We may assume that

(1) $G$ has no cutvertex.

For otherwise, $G$ is the union of two 2-edge-connected subgraphs $G_{1}, G_{2}$ having precisely one vertex $v$ in common. The minimality condition (i) implies that each of $G_{1}, G_{2}$ has the desired edge-coloring. By permuting colors in one of them, if necessary, also the edges incident with $v$ satisfies the conclusion of the theorem.

(2) Each vertex of $G$ has degree divisible by 3 . 
For, if $v$ has degree 1 modulo 3 , then we delete two edges $v v_{1}, v v_{2}$, we add a new vertex $v^{\prime}$ and the three edges $v^{\prime} v, v^{\prime} v_{1}, v^{\prime} v_{2}$. If $v$ has degree 2 modulo 3 , then we subdivide an edge $v v_{1}$ by inserting a new vertex $v^{\prime \prime}$ of degree 2 on the edge, and then we add an edge $v v^{\prime \prime}$ so that $v$ gets degree divisible by 3 . Then we apply the minimality condition (ii) to the new graph so that the new graph, and hence also $G$, satisfies the conclusion of Theorem 1 .

(3) $G$ has at least one vertex of degree $>3$.

For otherwise, the 4-color theorem gives a contradiction.

(4) $G$ is 3-edge-connected.

For, if $e_{1}=x_{1} x_{2}, e_{2}=y_{1} y_{2}$ are two edges such that $G-e_{1}-e_{2}$ has two components $G_{1}, G_{2}$ where $G_{1}$ contains $x_{1}, y_{1}$, then we apply the minimality condition (iv) of $G$ to $G_{1}+x_{1} y_{1}$ and $G_{2}+x_{2} y_{2}$, respectively.

If $x$ is a vertex of degree $>3$, then we select three consecutive edges around $x$, we delete the edges from $x$ and rename $x$ by $x^{\prime}$. We add a new vertex $x^{\prime \prime}$, we put the three deleted edges back and let them be incident with $x^{\prime \prime}$. We say that $x$ has been split into $x^{\prime}, x^{\prime \prime}$ and we may assume

(5) the new graph has a vertex $y$ distinct from $x^{\prime}, x^{\prime \prime}$ such that each path from $x^{\prime}$ to $x^{\prime \prime}$ contains $y$.

For otherwise, the new graph is 2-connected, it has the same number of edges as $G$, but the sum of degrees $>3$ is smaller. Now the minimality condition (iii) proves (5).

Note that $G-x-y$ is disconnected. We call $x, y$ a 2-vertex-cut.

(6) If a vertex $x$ of degree $>3$ is joined to a vertex $y$ by two or more edges, then $x, y$ is a 2 -vertex-cut.

For we can split $x$ into $x^{\prime}, x^{\prime \prime}$ such that $y$ is a common neighbor of $x^{\prime}, x^{\prime \prime}$. Now (6) follows from (5).

(7) Every vertex has at least three distinct neighbors.

For, if $x$ has only two neighbors $y, z$, then we may assume that $x$ is joined to $y$ by at least two edges. By (1), $G-y$ is connected. Hence also $G-y-x$ is connected. By (4), at least one of $x, y$ has degree $>3$. This contradiction 
to (6) proves (7).

Now we choose a 2 -vertex-cut $x, y$ such that the smallest component $G^{\prime}$ of $G-x-y$ is smallest possible and such that at least one of $x, y$ has degree $>3$. By (7), $G^{\prime}$ has at least two vertices.

(8) $G$ has at least two edges from each of $x, y$ to $G^{\prime}$.

Assuming that $x$ has degree $>3$, the minimality of $G^{\prime}$ implies that $G$ has at least two edges from $y$ to $G^{\prime}$. If $G$ has only one edge from $x$ to a vertex $x^{\prime}$ in $G^{\prime}$, then we may assume that $y$ has degree $>3$ since otherwise, $G$ would have an edge-cut with only two edges, contradicting (4). But now the 2 -vertex-cut $y, x^{\prime}$ contradicts the minimality of $G^{\prime}$. This proves (8).

(9) We may choose $x$ to have degree $>3$, and $G$ has at least two edges from $x$ to $G-V\left(G^{\prime}\right)-y$.

For, if $G$ has only one such edge, then (4) implies that $G$ has at least two edges from $y$ to $G-V\left(G^{\prime}\right)-x$. Combining this with (8) we conclude that $y$ has degree $>3$. Now (9) follows by letting $x, y$ interchange roles.

Now we split $x$ into $x^{\prime}, x^{\prime \prime}$ as follows: If there are precisely two edges from $x$ to $G^{\prime}$, then there is precisely one edge from $x^{\prime}$ to $G^{\prime}$ and precisely one edge from $x^{\prime \prime}$ to $G^{\prime}$. If there are more than two edges from $x$ to $G^{\prime}$, then there are precisely two edges from $x^{\prime \prime}$ to $G^{\prime}$. Then there is a path between $x^{\prime}, x^{\prime \prime}$ whose intermediate vertices all are in $G^{\prime}$, and there is another path between $x^{\prime}, x^{\prime \prime}$ that does not intersect $G^{\prime}$. This contradicts (5) and shows that the counterexample $G$ does not exist.

\section{Orientations and factorizations}

Jaeger [4], [5] generalized Tutte's 3-flow conjecture to the following conjecture which has become known as the the circular flow conjecture: If $k$ is an odd natural number, and $G$ is a $(2 k-2)$-edge-connected graph, then $G$ has an orientation such that each vertex has the same indegree and outdegree modulo $k$. The conjecture has recently been proved to be false [3], except possibly for $k=3,5$, but the following version was proved in [12]: Let $k$ be a natural number, and let $G$ be a $\left(2 k^{2}+k\right)$-edge-connected graph with $n$ 
vertices $v_{1}, v_{2}, \ldots, v_{n}$. Let $d_{i}$ be an integer for each $i=1,2, \ldots, n$ such that the sum of all $d_{i}$ is congruent modulo $k$ to the number of edges of $G$. Then $G$ has an orientation such that each $v_{i}$ has outdegrees $d_{i}$ modulo $k$. Theorem 3.1 in [7] gives the same conclusion under the weaker assumption that the quadratic bound $2 k^{2}+k$ is replaced by the linear bound $3 k-3$ when $k$ is odd. And if $k$ is odd and we seek a balanced orientation modulo $k$ (that is, every vertex gets the same indegree and outdegree modulo $k$ ), then it suffices that the odd edge-connectivity is at least $3 k-2$ by Theorem 4.12 in [7]. The result was reformulated as a result about factors modulo $k$ in [13]. Perhaps more surprisingly, we point out that the trick used for group flow in [13] can also be applied to factors (without reducing modulo $k$ ).

If $r$ is even, then every $r$-regular graph can be edge-decomposed into 2factors and hence into $q$-factors whenever $q$ is even and divides $r$. We shall now decompose an $r$-regular graph into $q$-factors where $q$ is odd and divides $r$.

Theorem 2 Let $r$ be a natural number $\geq 4$ and let $G$ be an r-regular graph of even order. Let $r=k q$ be a natural number where $q \geq 3$ is odd. If $k$ is odd and $G$ has odd edge-connectivity at least $3 k-2$, then $G$ can be edgedecomposed into $q$-factors. If $k$ is even and $G$ has an even number of vertices and edge-connectivity at least $k^{2}+2 k$, then $G$ can be edge-decomposed into $q$-factors.

Proof of Theorem 2: Assume first that $k$ is odd. By Theorem 4.12 in [7], the edges in $G$ can be oriented such that every vertex is balanced modulo $k$. As every degree is divisible by $k$, this implies that every vertex has indegree and outdegree divisible by $k$. Now split every vertex $v$ of positive outdegree and positive indegree in $G$ into two vertices $x_{1}, x_{2}$ such that each edge entering $x$ now enters $x_{1}$, and each edge leaving $x$ now leaves $x_{2}$. The resulting graph $G_{1}$ is bipartite, and every vertex has degree divisible by $k$. We split every vertex of $G_{1}$ into vertices of degree precisely $k$. The resulting graph $G_{2}$ is bipartite and $k$-regular and is therefore the union of $k 1$-factors. Each 1-factor in $G_{2}$ is a $q$-factor in $G$.

Assume next that $k$ is even. By Theorem 2 in [12], the edges in $G$ can be oriented such that every vertex has outdegree 0 modulo $k$. (Here we use the assumption that $G$ has an even number of vertices, because the sum of prescribed outdegrees, which is 0 modulo $k$, must be congruent to the number of edges of $G$ modulo $k$.) Now repeat the proof of the case where $k$ is odd. 
The reason for the difference in the connectivity bounds $3 k-2$ and $k^{2}+2 k$, in Theorem 2 when $k$ is odd, respectively even, is that $k$ is allowed to be even in [12] whereas $k$ is assumed to be odd throughout [7]. (In particular, Theorem 1.11 in [7] is stated in weaker form than actually proved in [12].) If the afore-mentioned Theorem 3.1 in [7] can be proved also for $k$ even, then $k^{2}+2 k$ in Theorem 2 can be replaced by $3 k-3$ provided we also replace 'odd edge-connectivity' by 'edge-connectivity'. To see that such an extension would be of interest in the present context, let us consider a 9regular graph of odd edge-connectivity at least 7 or a 15-regular graph of odd edge-connectivity at least 13 . By Theorem 2 such a graph has an edgedecomposition into 3-factors. But, we do not know if every 12-regular, 12edge-connected graph of even order has an edge-decomposition into 3-factors, see also Conjecture 2 below.

If $r=9, k=q=3$, then the required odd edge-connectivity in Theorem 2 is 7 . This can be reduced to 5 if Tutte's 3 -flow conjecture holds. Indeed, the 3 -flow conjecture says that any graph with odd edge-connectivity $>3$ has an orientation which is balanced modulo 3 .

Theorem 3 Let $r$ be an odd natural number divisible by 3. Let $G$ be an $r$ regular graph of odd edge-connectivity at least $r-2$. Let $r=r_{1}+r_{2}+\cdots+r_{m}$ be any partition of $r$ with all parts $r_{i} \geq 2$. Then $G$ can be edge-decomposed into subgraphs $G_{1}, G_{2}, \ldots, G_{m}$ such that $G_{i}$ is an $r_{i}$-factor for $i=1,2, \ldots, m$.

Proof of Theorem 3: By Theorem 2, G is the union of $r / 3$ 3-regular graphs $G_{1}, G_{2}, \ldots, G_{r / 3}$. Assume $r_{1}, r_{2}, \ldots, r_{p}$ are odd and $r_{p+1}, r_{p+2}, \ldots, r_{m}$ are even. The union of $G_{p+1}, G_{p+2}, \ldots, G_{r / 3}$ is regular of even degree and can therefore be edge-decomposed into 2-factors. We use these 2-factors to extend $G_{1}, G_{2}, \ldots, G_{p}$ to graphs that are regular of the desired odd degrees, and to construct the remaining $G_{p+1}, G_{p+2}, \ldots, G_{m}$.

Theorem 4 Let $r$ be any odd natural number divisible by 3, and let $G$ be any $(r-3)$-edge-connected graph of even order and maximum degree at most $r$. Let $r=r_{1}+r_{2}+\cdots+r_{m}$ be any partition of $r$ with all parts $r_{i} \geq 2$. Then $G$ can be edge-decomposed into spanning subgraphs $G_{1}, G_{2}, \ldots, G_{m}$ such that $G_{i}$ has maximum degree at most $r_{i}$ for $i=1,2, \ldots, m$.

Proof of Theorem 4: We add as many edges as possible so that the resulting graph $G^{\prime}$ has maximum degree at most $r$. If $G^{\prime}$ is $r$-regular we 
apply Theorem 3. Otherwise, $G^{\prime}$ has precisely one vertex $u$ of degree $<r$. Since $G$ has even order, the degree of $u$ has the same parity as $r$. As $G$ has odd edge-connectivity at least $r-2, u$ has degree $r-2$ in $G^{\prime}$, and all other vertices have degree $r$. We orient the edges incident with $u$ such that $u$ is balanced modulo $k=r / 3$. Again, by Theorem 4.12 in [7], the remaining edges in $G^{\prime}$ can be oriented so that each vertex is balanced modulo $k$. As in the proof of Theorem 2 we split every vertex into a source and a sink. We add a directed edge from the source arising from $u$ to the sink arising from $u$. We split every vertex of degree $2 k$ or $3 k$ into vertices of degree precisely $k$. As the resulting graph is bipartite and $k$-regular we complete the proof as in Theorem 2.

Theorem 5 Let $r$ be any odd natural number divisible by 3, and let $G$ be any $(r-3)$-edge-connected graph such that each vertex has odd degree at least $r$. Let $r=r_{1}+r_{2}+\cdots+r_{m}$ be any partition of $r$ with all parts $r_{i} \geq 2$. Then $G$ can be edge-decomposed into spanning subgraphs $G_{1}, G_{2}, \ldots, G_{m}$ such that $G_{i}$ has minimum degree at least $r_{i}$ for $i=1,2, \ldots, m$.

Proof of Theorem 5: The proof is by induction on the number of edges. If $G$ has only two vertices and $r$ edges, there is nothing to prove. So we proceed to the induction step.

If $G$ is $r$-regular (and hence of odd edge-connectivity at least $r-2$ ) we apply Theorem 3. If $G$ has a vertex $v$ of degree at least $r+2$ and with at least two neighbors, then we use Mader's lifting theorem [8] which says that we can lift two edges $v x, v y$ which means that we replace those two edges with only one edge $x y$ such that the resulting graph is $(r-3)$-edge-connected. We complete the proof by applying the induction hypothesis to that graph. If $v$ has only one neighbor $u$ then we delete $v$ and apply Mader's lifting theorem to lift the remaining edges incident to $u$ so that the resulting graph is $(r-3)$-edge-connected. Then we complete the proof by induction.

The reason that we use edge-connectivity and not odd edge-connectivity in Theorem 5 is that Mader's lifting does not apply to odd edge-connectivity.

\section{Open problems}

If $G$ is an $r$-regular graph of even order and odd edge-connectivity at least $r-2$, and $r$ is odd and divisible by a number $q \geq 3$, then the proof of Theorem 
3 shows that the conclusion of that theorem holds if each part $r_{i} \geq q-1$. We believe the following stronger statement holds.

Conjecture 1 Theorem 3 also holds when $r$ is not divisible by 3.

Most of our results assume that $r$ is odd. In Conjecture 2 below $r$ may be even. Then we must add the condition that the graph has an odd number of vertices.

Conjecture 2 Let $G$ be an $r$-regular $(r-2)$-edge-connected graph of even order. Then $G$ can be edge-decomposed into factors such that all, except possibly one, are 3-factors, and the remaining factor is a 2-factor or a 4factor.

In Theorems 3,4,5 it is necessary to require that all $r_{i} \geq 2$ since there are $(r-2)$-edge-connected $r$-regular graphs of even order but with no 1-factor. If we raise the edge-connectivity to $r-1$ or $r$ we may possibly allow some $r_{i}$ to be 1 . For dense regular graphs without multiple edges this problem has attracted much attention culminating with the deep 1-factorization result by Csaba, Kühn, Lo, Osthus and Treglown [2]. Seymour's version of Goldberg's conjecture (see [6] page 192) implies that any $r$-regular graph of even order and odd edge-connectivity at least $r-1$ has chromatic index (edge-chromatic number) at most $r+1$. If $r$ is large compared to the order, this implies that many of the color classes are 1-factors.

Problem 1 Is every $r$-regular, r-edge-connected graph of even order the union of $r-2$ 1-factors and a 2-factor?

An affirmative answer to Problem 1 would at the same time prove Seymour's conjecture and also essentially prove the 1-factorization result in [2]. For $r=9$ it would imply that every 3-regular 3-connected graph has seven 1-factors and a 2-factor that cover each edge thrice. In particular, the seven 1factors cover the edges of the graph. This comes close to the Berge-Fulkerson conjecture (see [1] page 250 and [6] page 192) which implies (and is in fact equivalent to, see [9]) that there are five such 1-factors. So it may be too much to expect an affirmative answer to Problem 1. Instead we propose the following much weaker statement: 
Conjecture 3 There exists a natural number $r_{0}$ such that, for every $r \geq$ $r_{0}$, every r-regular, r-edge-connected graph of even order has two disjoint 1-factors.

The snarks show that $r_{0} \geq 4$ and so the 4-regular case is particularly interesting. In this case Nash-Williams made the stronger conjecture that every 4-regular 4-connected graph is Hamiltonian. As observed by Meredith [10], if we double the edges in a 1-factor of the Petersen graph, then the resulting 4-regular 4-edge-connected graph has chromatic index 5, and Meredith used this to disprove Nash-Williams' conjecture, see [1] page 239. The Meridith construction also shows that Conjecture 3 is equivalent with the conjecture where ' $r$-edge-connected graph' is replaced by ' $r$-connected simple graph'.

It may be useful to first address the even weaker question below.

Conjecture 4 If $r$ is an odd natural number $\geq 5$, then every $r$-regular, $r$ edge-connected graph is the union of three regular factors each of odd degree.

For $r \geq 9$, this would follow from Conjecture 1. For $r=5$ it is equivalent to the statement in Conjecture 3 (when restricted to $r=5$ ). By Theorem 2, Conjecture 4 holds for each $r \geq 9$ that is not a prime.

\section{References}

[1] J. A. Bondy and U. S. R. Murty, Graph Theory with Applications. The MacMillan Press Ltd. (1976).

[2] B. Csaba, D. Kühn, A. Lo, D. Osthus and A. Treglown, Proof of the 1-factorization and Hamilton decomposition conjectures II, arXiv:1401.4164v2 [math.CO] 23.Oct.2014.

[3] M. Han, J. Li, Y. Wu, C.-Q. Zhang, Counterexamples to Jaegers Circular Flow Conjecture, J. Combinatorial Theory, Ser B 131 (2018) 1-11.

[4] F. Jaeger, On circular flows in graphs. Proc.Colloq. Math. János Bolyai 37(1982) 391-402.

[5] F. Jaeger, Nowhere-zero flow problems. In: Selected Topics in Graph Theory 3 edited by L. W. Beineke and R. J. Wilson. Academic Press (1988) 71-95. 
[6] T. Jensen and B. Toft, Graph Coloring Problems. John Wiley, New York (1995).

[7] L.M. Lovász, C. Thomassen, C. Q. Zhang and Y. Wu, Nowhere-zero 3-flows and modulo k-orientations, J. Combinatorial Theory, Ser B 103 (2013) 587-598.

[8] W. Mader, A reduction method for edge-connectivity in graphs, Ann. of Discrete Math. 3 (1978) 145-164.

[9] G. Mazzuoccolo, The equivalence of two conjectures of Berge and Fulkerson, J. Graph Theory 68 (2011) 125-128.

[10] G.H.J. Meredith, Regular $n$-valent $n$-connected nonhamiltonian non- $n$ edge-colorable graphs, J. Combinatorial Theory, Ser B 14 (1973) 55-60.

[11] B. Mohar and C. Thomassen, Graphs on Surfaces. Johns Hopkins University Press (2001).

[12] C. Thomassen, The weak 3-flow conjecture and the weak circular flow conjecture, J. Combinatorial Theory, Ser B 102 (2012) 521-529.

[13] C. Thomassen, Graph factors modulo k, J. Combinatorial Theory, Ser B 106 (2014) 574-177.

[14] C. Thomassen, Group flow, complex flow, unit vector flow, and the $(2+\epsilon)$-flow conjecture, J. Combinatorial Theory, Ser B 108 (2014) 8191.

[15] C. Q. Zhang, Integer flows and cycle covers of graphs. Marcel Dekker, Inc (1997). 